\title{
Discovering and Synthesizing Humanoid Climbing Movements
}

\author{
KOUROSH NADERI, JOOSE RAJAMÄKI, and PERTTU HÄMÄLÄINEN, Aalto University
}

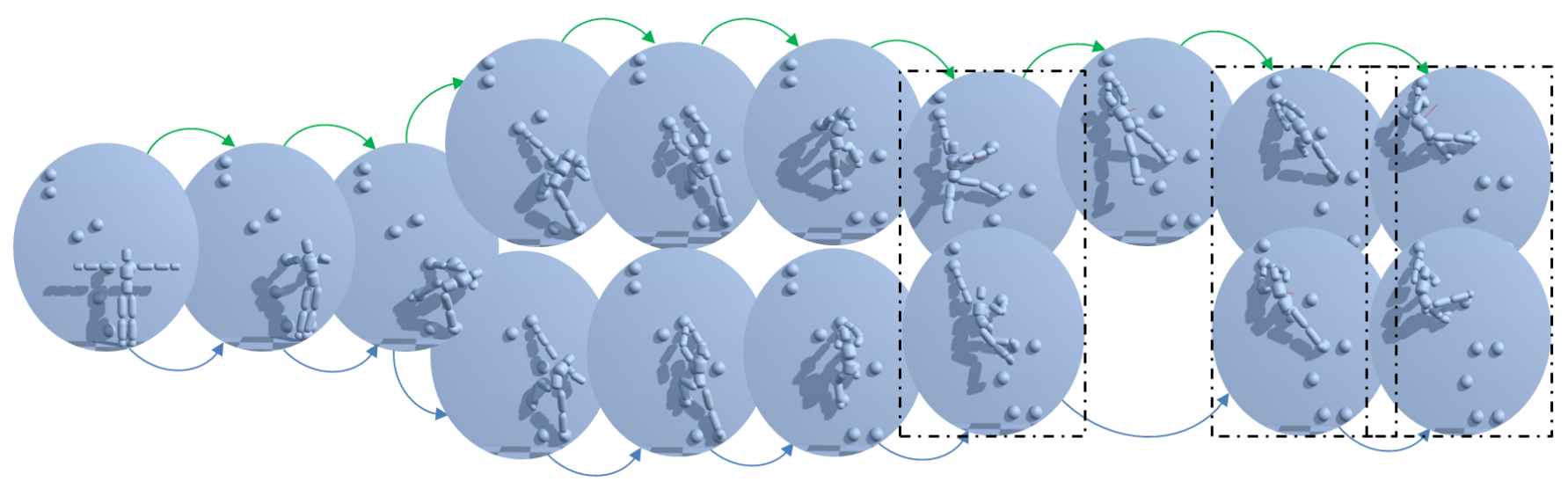

Fig. 1. Two paths (green and blue arrows) to the top hold in a bouldering problem, as discovered by our method. Compared to previous work, we are not limited to only moving one limb at a time or having the climber's hands and feet on predefined climbing holds; limbs can also hang free for balance, or use the wall for friction. The dashed rectangles highlight the one-to-many mapping from stances (assignments of holds to limbs) to climber states.

This paper addresses the problem of offline path and movement planning for wall climbing humanoid agents. We focus on simulating bouldering, i.e. climbing short routes with diverse moves, although we also demonstrate our system on a longer wall. Our approach combines a graph-based highlevel path planner with low-level sampling-based optimization of climbing moves. Although the planning problem is complex, our system produces plausible solutions to bouldering problems (short climbing routes) in less than a minute. We further utilize a k-shortest paths approach, which enables the system to discover alternative paths - in climbing, alternative strategies often exist, and what might be optimal for one climber could be impossible for others due to individual differences in strength, flexibility, and reach. We envision our system could be used, e.g. in learning a climbing strategy, or as a test and evaluation tool for climbing route designers. To the best of our knowledge, this is the first paper to solve and simulate rich humanoid wall climbing, where more than one limb can move at the same time, and limbs can also hang free for balance or use wall friction in addition to predefined holds.

\section{CCS Concepts: • Computing methodologies $\rightarrow$ Physical simulation;}

Additional Key Words and Phrases: Climbing Motion Synthesis, Rapidly Exploring Random Trees, K Shortest-Paths on Graph, Dynamic Graph, A $^{*}$ Prune

\section{Author's addresses:}

K. Naderi, e-mail: kourosh.naderi@aalto.fi

J. Rajamäki, e-mail: joose.rajamaki@aalto.fi

P. Hämäläinen, e-mail: perttu.hamalainen@aalto.fi

Department of Computer Science, Aalto University, Finland.

Permission to make digital or hard copies of all or part of this work for personal or classroom use is granted without fee provided that copies are not made or distributed for profit or commercial advantage and that copies bear this notice and the full citation on the first page. Copyrights for components of this work owned by others than ACM must be honored. Abstracting with credit is permitted. To copy otherwise, or republish, to post on servers or to redistribute to lists, requires prior specific permission and/or a fee. Request permissions from permissions@acm.org.

(c) 2017 ACM. 0730-0301/2017/7-ART43 \$15.00

DOI: http://dx.doi.org/10.1145/3072959.3073707

\section{ACM Reference format:}

Kourosh Naderi, Joose Rajamäki, and Perttu Hämäläinen. 2017. Discovering and Synthesizing Humanoid Climbing Movements. ACM Trans. Graph. 36, 4, Article 43 (July 2017), 11 pages.

DOI: http://dx.doi.org/10.1145/3072959.3073707

\section{INTRODUCTION}

Path/motion planning is crucial in many applications [Hoy et al. 2015; Rastgoo et al. 2014]. Motion planning for climbing humanoid agents is an intricate problem, and its applications vary from searchand-rescue in robotics [Bretl 2006] to simulation of virtual humanoid agent in computer games [Libeau et al. 2009]. Its difficulty arises from the high number of degrees of freedom (DoF) of the human body as well as the long planning horizon required. Even the lowlevel short horizon problem of fully physically based humanoid balancing while reaching for an object in any direction is a complex one, and efficient controllers for such tasks have only recently emerged [Hämäläinen et al. 2015; Jain et al. 2009; Tassa et al. 2014]. Planning a sequence of such complex movements is still to a large extent an unsolved problem. In climbing, one must consider collisions of the agent with the wall and itself as well as stability of balance; when switching holds, the agent may swing around or even fall. Finally, we are striving for physically plausible movement, i.e., using limited forces and torques that further constrain the movements.

In previous work, a variety of simplifications have been utilized. In his work on multi-limbed climbing robots, Bretl [2006] considered only 2 DoF per limb, and the robot's motions are planned in its configuration space and dynamically instable configurations are not allowed, which admits for a closed-form solution for the robot's configuration given its center and end effector positions. To implement human-like motions, we also need to consider full humanoid body dynamics, and since a closed form solution does not exist, 
an appropriate movement optimization method is needed. Such an approach is presented in [Jain et al. 2009], however focusing on single climbing moves without path planning. The agent also implements a "three-hold-rule", i.e., only moving one limb at a time and keeping others on climbing holds. Similar to other previous work in humanoid wall climbing, limbs are also restricted to only utilize predefined holds. However, research on human climbing suggests that climbers may prefer to use less than three holds at a time [Sibella et al. 2007]. In addition to standing on a hold, a foot can also be used on the wall for friction climbing, or a leg can hang in the air for balance, somewhat similar to how animals use their tails.

In this paper we are looking for an approach that can effectively utilize three or two holds at a time while using the free limbs for balancing or frictional contacts with the wall. Also, in order to simulate real climbing, we wish to find a feasible path from a starting stance that defines two allowed holds for the climber's hands to the so called "top hold" that can be reached with either hand, i.e., we also consider the agent's transition from standing to the wall. The only simplification we utilize is that we assume at least two holds to be used at the same time, in order to limit the size and connectivity of our search graph. We also focus on bouldering, a rapidly growing form of climbing on low walls without other safety equipment than a soft floor. Bouldering reduces the number of holds to consider, but is also perhaps more interesting from an animation perspective, as it tends to highlight complex, technical moves and climbing as a puzzle solving activity, as opposed to climbing long routes to build endurance or conquer one's fear of heights.

To summarize our technical approach, we grow a tree of paths in the climber's state space, inspired by Rapidly-Exploring Random Trees (RRT:s, [Lavalle 1998]). Planning in high-dimensional space using randomized actions can, however, take a long time and sometimes makes it impossible to find a feasible path [Garcia and How 2005]. Instead, we grow the tree informed by graph search in the lower-dimensional stance graph, which represents all possible 4-hold configurations or climber stances. Stances with free legs or hands are also allowed. At any time, an $A^{*}$ Prune search [Liu and Ramakrishnan 2001] of the stance graph gives us a path to try, and a sampling-based low-level controller is then utilized to optimize and simulate the transitions between stances. Even for a small bouldering route, the stance graph can have thousands of vertices and edges. However, we demonstrate that a set of edge and node preference rules based on observations of human climbers can guide the search so that the agent most of the time tries feasible paths first. If the low-level controller fails in a transition, the preference for the corresponding edge of the graph is adjusted, and $\mathrm{A}^{*}$ Prune is re-run to find an alternative path.

To the best of our knowledge, we are the first to demonstrate rich humanoid wall climbing with free limbs with a possibility to utilize wall friction, and to allow the user to browse alternative paths. Our system also works with balance-intensive routes, e.g., where all holds lie on a vertical line.

\section{RELATED WORK}

In this section, we first review some of the central research on path planning in high-dimensional spaces. We then continue on the more specific domain of humanoid climbing movements.

\subsection{Path Planning in High-Dimensional Space}

One of the most popular methods on navigation in high-dimensional state space is the artificial potential function (APF) [Guldner et al. 1997], which uses the information from both the environment and the robot for real-time path planning. However, it suffers from trapping into local minima, and thus there are many variations of this method trying to solve this problem. [Tanner and Kumar 2005] use the combination of a centralized method with APF to define a potential function for a multi-agent problem that navigates them in the environment without collisions. [Brock and Khatib 2002] introduce an elastic strip framework, which uses the combination of global path planning and local path planning. To satisfy global path planning, the initial and goal configurations are connected through a couple of traceable points by a local path planner, and APF is used as the local path planner to control the robot with high dimensional configuration space. Although there are some methods that deal with APF's problem of getting stuck in local minima, these methods are somewhat limited and need further information from the environment.

The emergence of sampling based methods like Rapidly-Exploring Random Tree (RRT) [Lavalle 1998] and Probabilistic Roadmaps (PRM) [Kavraki et al. 1996] has enabled global search without needing to encode environment information as a potential function. Both methods sample points in the agent's configuration space, and then connect the points to the previously sampled ones. PRM creates a graph that can be searched, e.g. using Dijkstra's method, whereas RRT produces a tree with the current or goal configuration as the root, and the tree provides a path to any sampled configuration. As the dimensionality of the search space increases, RRT needs more samples to find a feasible path, and performance decreases in cases where narrow passages exist in the space [Garcia and How 2005]. This is due to the fact that numerous samples fall in the highdimensional free space far from the narrow passages. To mitigate the dimensionality problems, RRT-Connect [Kuffner and LaValle 2000] attempts to greedily connect two trees, one growing from the start configuration, and the other from the goal configuration. RRT-Connect is faster than the original RRT [Lavalle 1998]; however, [Garcia and How 2005] improve the efficiency of RRT even more by using APF as the steering function that connects sampled points. [Garcia and How 2005] demonstrate the combination of RRTs with APF in controlling space crafts. The combination allows the path planner to grow a tree in the full state of multiple space crafts which has e.g. 48 DOF for 8 space crafts.

Our approach resembles RRT, as we grow a tree with a standing pose near the wall as the root. However, although some RRT variants can handle limited dynamics [LaValle and Kuffner 2001; Webb and van den Berg 2013], RRTs alone are not enough to solve climbing humanoid movement with simulated dynamics in addition to wall and self-collisions.

\subsection{Motion Planning for Climbing Humanoid Agents}

An investigation about motion planning of a multi-limbed robot has been carried out by [Bretl 2006]. The method assumes that each limb of the robot has 2 DOF. The robot stances are kept in a priority queue and the algorithm explores them by sampling a 
robot stance from the queue and adding adjacent stances to the sampled one. Adjacent robot stances are explored between holds by solving for the closed form solution of the robot's configuration given the robot's center and end limbs positions. When final desired robot stance is found, the method uses PRM to explore the robot's configuration space for a detailed path to the stance. Sampled robot configurations are accepted when they satisfy joint limitations and the robot's static stability. However, no closed form solution exists for limbs with more than $3 \mathrm{DOF}$, and each limb of our simulated humanoid agent has 4 controlled DOFs (shoulder 3 and elbow 1). [Libeau et al. 2009] use inverse kinematics inspired by gradient descent to implement the transition between different climber's stances where each climber stance includes four hold positions. They use a reinforcement learning method to learn which sequence of climber's stances eventually lead the agent to the top of the wall. They enable the humanoid agent to climb a wall by reusing what has already been learnt from previous experience. However, using inverse kinematics to find the transition between climber's stances can confine the diversity of behaviors that emerge from the climber, and lead to unnatural looking motions on the planned path.

[Tonneau et al. 2014] use climbing as a test case for their method for generating contacts and poses for exerting force in a userspecified direction. Motion is, however, synthesized using an IKbased approach without dynamics. [Olsen et al. 2014] build on this in their impressive simulation of a humanoid with full finger articulation grasping and hanging from climbing holds. Our work complements [Olsen et al. 2014] in that we assume a simpler hold model and instead focus on the path planning and full-body motion synthesis of complete climbing routes. In the future, we naturally hope to integrate both approaches.

In particular, our work is inspired by the optimization-based humanoid climbing movements of [Hämäläinen et al. 2015; Jain et al. 2009; Mordatch et al. 2012], and the graph-based climbing stance path search of [Dung and Shimada 2014]. The former have shown that optimization of full-body humanoid movement control with respect to a cost function can lead to the emergence of climbing movements, although longer sequences of such movements were not demonstrated. [Dung and Shimada 2014] on the other hand build a graph of climbing robot stances, and find the best path on the static graph using Dijkstra's algorithm. Such a stance graph can also be considered a generalization of footprint-based roadmaps of [Choi et al. 2011, 2003]. Each node in the graph of [Dung and Shimada 2014] contains four hold positions for robot's arms and feet, and graph building is done by considering all possible combinations of four holds reachable by the robot's arms and feet given different reference points on the wall. The nodes that represent adjacent robot stances, i.e. only one hold position differs, are connected by an edge. The algorithm only focuses on the path planning on the stance graph where the costs on the graph edges are fixed, and it is assumed that the best path returned by Dijkstra's algorithm can be followed by the humanoid robot; here, moving only a single limb at a time helps due to the three fixed limbs providing stable balance.

In this paper, we combine climbing movement optimization in the style of [Hämäläinen et al. 2015; Jain et al. 2009; Mordatch et al. 2012] with the stance graph search of [Dung and Shimada 2014]. This allows our agent to plan and execute long sequences of rich, dynamic climbing movements. Extending [Dung and Shimada 2014], we allow the agent to move more than one limb at a time leading to more dynamic movements, and explore multiple shortest paths in order to allow comparing them, e.g. finding the one that required least effort in the optimized climbing movements. We also allow failed moves through the use of a dynamic graph edge costs; after a failure the cost of an edge is increased to make the graph search favor alternative paths.

Outside animation and motion synthesis, technological augmentations of climbing have been investigated, e.g., by instrumenting a bouldering wall with sensors [Aladdin and Kry 2012] and enabling interactive challenges and user-designed climbing problems with computer vision and projected graphics [Kajastila et al. 2016].

\section{PRELIMINARIES: K-SHORTEST PATHS IN A DYNAMIC GRAPH}

As explained above, we are finding multiple paths in the stance graph, and the graph edge costs may change dynamically. Thus, the problem falls in the category of "dynamic shortest paths" or "shortest paths in a dynamic graph". However, instead of finding shortest paths from one graph node to all other nodes, we want $k$-shortest paths between two terminal nodes. A good review on methods for the shortest paths problem can be found in [Eppstein 1998; Eppstein et al. 2016]. In this paper we utilize $A^{*}$ prune [Liu and Ramakrishnan 2001], a general algorithm for returning $k$ shortest paths between two terminal graph nodes. The method is based on the original $\mathrm{A}^{*}$; however, instead of keeping two lists, it only keeps one list of all possible paths to a graph node which is sorted based on heuristics costs (see Section 5.2).

[Nannicini and Liberti 2008; Roditty and Zwick 2004] provide reviews for shortest paths methods on dynamic as opposed to static graphs. There exists methods that replan a path after some changes happen to the graph [Likhachev et al. 2005], however there is no general way to return $k$-shortest paths between two terminal graph nodes after a graph alteration. Our stance graphs are large, but their connectivity is fortunately sparse enough to allow simply running $A^{*}$ prune again after changing graph edge costs.

\section{NOTATION AND PROBLEM DEFINITION}

Environment: Fig 2 shows climbing humanoid agent in 3D environment where the global $\vec{z}$ and $\vec{x}$ axes are upwards and to the right, respectively. The climbing wall and holds are usually in $x z$ surface, however in some cases the wall is tilted (see Section 6). We denote positions with $\mathbf{x} \in \mathbb{R}^{3}$.

We have a set of holds, denoted by $\mathcal{H}$, containing $N$ different hold positions represented by $\mathbf{x}_{h}$ for $h=1 \ldots N$. The holds are simulated as ball-and-socket joints. The limb-hold joints do not have motors; the character is thus underactuated and requires balancing when less than 3 holds are used. The hold denoted by $\mathbf{x}_{g}$ is the goal hold to be grasped by either of the climber's hands, and it is usually located on the top of the wall. The climber starts from the ground where its hands and feet are detached from holds, and has to first get its hands on user-defined starting holds and then reach $\mathbf{x}_{g}$. During climbing, the agent is also allowed to use the wall in addition to the holds (as usual in indoor climbing); however, we only plan the paths 


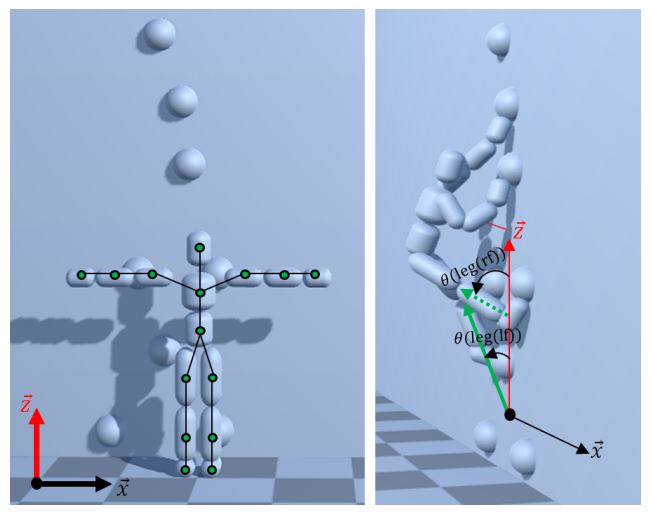

Fig. 2. Left: Humanoid Body Skeleton. Right: Angles of climber's legs with $\vec{z}$ axis.

using the holds and possible wall usage emerges from movement optimization if no target hold is specified for a limb.

Humanoid Climbing Agent: Let us denote the full climbing humanoid state with $\mathcal{S}$. $\mathbf{s} \in \mathcal{S}$ contains $\left[\mathbf{x}_{i}, \mathbf{q}_{i}, \mathbf{v}_{i}, \omega_{i}\right]$ for $i=1 . . m$ where $m$ is the number of bones in the humanoid agent, and $\mathbf{x}_{i}, \mathbf{q}_{i}, \mathbf{v}_{i}$ and $\omega_{i}$ are position, rotation, linear velocity and angular velocity of each bone, respectively. Each bone of the humanoid agent is named, and its end position is denoted by $\mathbf{x}_{\{\text {bone name }}$, e.g. $\mathbf{x}_{\text {trunk }}$ denotes the trunk's position. The positions of climber's hands and feet are noted by $\{$ lf,rf,lh,rh $\}$, which stand for left and right feet, and left and right hands, respectively. In addition, $\mathcal{S}$ contains information on whether the climber's hands and feet are connected to the holds or not.

Climbing Stance and Connectivity: In addition to the full climber state $\mathbf{s}$, our method utilizes the concept of a stance, which is defined as an assignment of holds to the climber's hands and feet, without considering body pose. To mitigate the problems of high state space dimensionality, we first plan the path in the stance space, and utilize the results to set goals for the state space planning.

We denote a climber's stance with $\sigma$, and the high-level path planner plans a sequence of stances from starting posture towards the goal hold. $\sigma_{i}=\left[\mathrm{x}_{\mathrm{lf}}, \mathrm{x}_{\mathrm{rf}}, \mathrm{x}_{\mathrm{lh}}, \mathrm{x}_{\mathrm{rh}}\right]$ denotes left foot, right foot, left hand and right hand hold positions reached or to be reached, respectively. Each element of $\sigma_{i}$, denoted by $\sigma_{i, j}$ where $j=\{$ lf,rf,lh,rh $\}$, can be either $\mathbf{x}_{-1}$ or $\mathbf{x}_{h} \in \mathcal{H}$. We define $\mathbf{x}_{-1}$ as free position or not attached notation since we want to allow the climber to have a free hand or foot while climbing. The agent starts at $\mathbf{s}_{0}$ from free stance $\sigma_{0}=\left[\mathbf{x}_{-1}, \mathbf{x}_{-1}, \mathbf{x}_{-1}, \mathbf{x}_{-1}\right] . \sigma_{i}$ stance is a goal stance if $\sigma_{i, \text { rh }}$ or $\sigma_{i, \text { lh }}$ equals $\mathbf{x}_{g}$, and thus if we have $n$ different goals, we denote them by $\sigma_{g 1} \ldots \sigma_{g n}$. We use the notation of $\sigma_{g}$ in the path planned by the high-level path planner, denoted by $p\left(\sigma_{0}, \sigma_{g}\right)$, as a goal stance that is the first to be reached by the high-level path planner from $\sigma_{0}$. The path should start from $\sigma_{0}$ and go to $\sigma_{g}$ through a user-defined $\sigma_{\text {start }}$.

The planned path of $p\left(\sigma_{0}, \sigma_{g}\right)$ is used to grow a tree denoted by $\mathcal{T}$ in the high-dimensional climber's state space with the help of the low-level controller. Let us assume $s$ is a state in $\mathcal{T}$. We denote the stance that the climber has reached at state $\mathbf{s}$ by $\sigma_{\mathbf{s}}$. Then, if $\sigma_{\mathrm{s}}$ equals $\sigma_{i}$ in the planned path, we want to simulate forward the climber's state from $\mathbf{s}$ towards $\sigma_{i+1} \in p\left(\sigma_{0}, \sigma_{g}\right)$ with the help of the low-level controller in order to follow the planned path. To reach $\sigma_{i+1}$ from $\sigma_{\mathbf{s}}$, the agent first has to let go of the holds that differ from $\sigma_{i+1}$. Then, the low-level controller can try to get the climber's hands and feet to $\sigma_{i+1}$. The climber has reached $\sigma_{i+1}$ if the following conditions are satisfied.

- For $j=\{\mathrm{lh}, \mathrm{rh}\}: \sigma_{\mathrm{s}, j}=\sigma_{i+1, j}$ if $\left\|\mathbf{x}_{j}-\sigma_{i+1, j}\right\|<r_{h}$

- For $j=\{$ lf,rf $\}: \sigma_{\mathrm{s}, j}=\sigma_{i+1, j}$ if $\left\|\mathbf{x}_{j}-\sigma_{i+1, j}\right\|<r_{h}$ and $\theta(\operatorname{leg}(j))<\theta_{0}$

where $r_{h}$ and $\theta_{0}$ are distance and angle thresholds for reaching conditions of hands and feet, respectively. The angle threshold is only used in reaching conditions of the feet, and $\theta(\operatorname{leg}(j))$ is calculated as $\angle(\vec{z}$, $\operatorname{dir}(\operatorname{leg}(j)))$ and denotes the angle between the shin of leg $j$ with global $\vec{z}$ (see Fig 2). If the conditions are not satisfied, the lowlevel controller has failed to get the climber's hands and feet to $\sigma_{i+1}$ on the planned path, and the hands and feet that got disconnected in $\sigma_{\mathrm{s}}$ remain disconnected, and thus we reach another stance on the stance graph instead of the planned $\sigma_{i+1}$ (see Fig 3c).

\section{MOTION PLANNING FOR A CLIMBING AGENT}

On a high level, our approach entails building the stance graph, followed by an iteration of 1) finding a yet non-simulated shortest path in the graph based on edge cost heuristics, 2) optimizing and simulating the physically based movements corresponding to path edges, i.e., stance-to-stance climbing moves, and 3) updating graph edge costs if step 2 fails. The last step causes the graph search to return alternative paths, although if there are several failures, failed moves will eventually be tried again.

The method is presented as pseudocode in Algorithm 1, and illustrated in Fig 3. As shown in Fig 3d, the end result is a tree of realized movements in the climber's state space, where one or more tree vertices can map to the same stance graph vertex, but tree edges map uniquely to stance graph edges. In effect, the stances can be considered points in a low-dimensional subspace of the full state.

We now first go through the algorithm, and then explain the phases of graph building, path finding, and the low-level optimization of climbing moves in respective subsections.

We initialize the tree at $s_{0}$ in line 3 and build the stance graph of $\mathcal{G}_{\sigma}$ in line 4 (Fig 3a). Section 5.1 explains the details of this process. The costs for stance vertices and transitions among them are defined such that the most a-priori preferred path on the stance graph has the lowest cost. To grow the tree, we use $A^{*}$ prune [Liu and Ramakrishnan 2001] in line 7 to find yet not simulated paths from the stance graph. We then use either C-PBP and CMA-ES, two different physically based sampling/optimization methods to optimize and simulate the moves corresponding to each not yet simulated stance path edge. Details are explained in Section 5.3.

Fig 3 b illustrates a case where the low-level controller successfully grows the tree from its starting tree node on the planned path. Thus, all end states $\mathbf{s}_{\text {end }}$ are added to the tree in line 19, and climber's stance at the end of the forward simulation or $\sigma_{\text {end }}$ is at target stance or $\sigma_{i+1}$ (Line 22). In contrast, Fig 3c shows a case of the lowlevel controller failing to grow the tree toward $\sigma_{i+1}$. In this case, the node will be added to the tree in a different stance than $\sigma_{i+1}$, which 


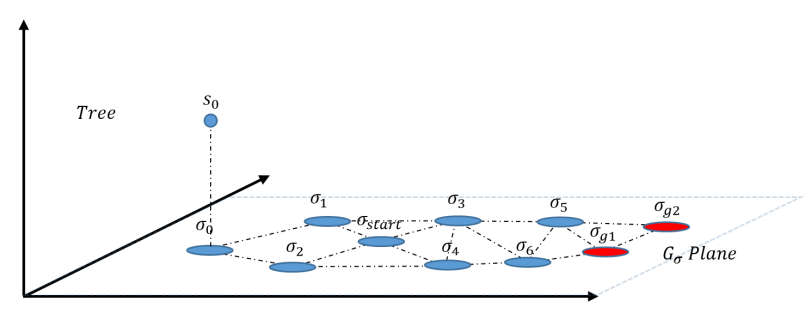

(a) Initialization of tree root of $\mathrm{s}_{0}$ and stance graph of $\mathcal{G}_{\sigma}$.

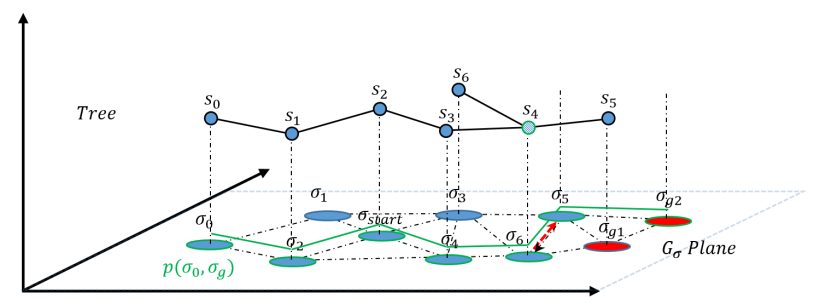

(c) Second case: the low-level controller failed to grow the tree on the second sampled path from $\mathbf{s}_{4}$ toward $\sigma_{5}$ and instead it goes to $\sigma_{3}$ at $\mathbf{s}_{6}$. The transition cost for $\sigma_{6}$-to- $\sigma_{5}$ is increased, and now has a higher cost than $\sigma_{5}$-to- $\sigma_{6}$.

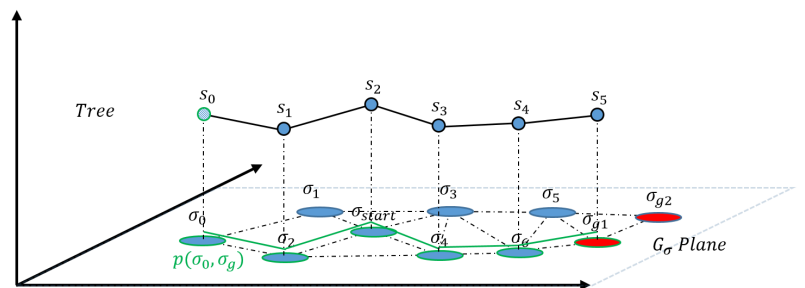

(b) First case: the low-level controller successfully grows the tree on the sampled path of $p\left(\sigma_{0}, \sigma_{g}\right)$ from the stance graph.

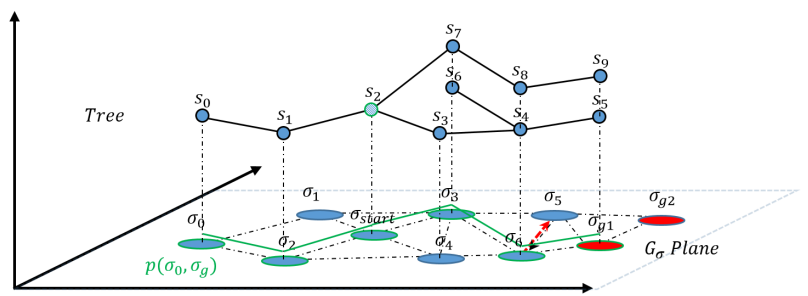

(d) Third case: the low-level controller successfully grows the tree on the third sampled path starting from $\mathbf{s}_{2}$. Although there are tree nodes representing $\sigma_{6}$ and $\sigma_{g 1}$, new tree nodes are added in growing process.

Fig. 3. Various stages of Algorithm 1. The xz-plane denotes the stance dimensions, and vertical axis denotes the additional dimensions of climber body state s. Our method grows a tree in the state space (in this abstract figure the $3 D$ space) guided by the lower-dimensional stance graph (here the xz-plane). As illustrated in subfigure (d), a single stance may map to multiple states, but tree edges uniquely map to stance graph edges. All paths start from $\sigma_{0}$ and pass through user-specified stance $\sigma_{\text {start }}$. There are two goal stances in the graph denoted by $\sigma_{g 1}$ and $\sigma_{g 2}$. The starting tree node for growing the tree for each planned path is denoted by green color. The planned path of $p\left(\sigma_{0}, \sigma_{g}\right)$ is denoted by green solid lines above the stance graph. Red dashed edges in the graph denote increased edge cost after failure.

may have a limb free instead of assigned to the planned target hold (Line 18). In this case, we increase the transition cost from $\sigma_{i}$ to $\sigma_{i+1}$ on the graph (Line 25), and stop growing tree (Line 26) since we have deviated from the planned path. It should be noted that the transition cost from $\sigma_{i+1}$ to $\sigma_{i}$ remains same since a transition from $\sigma_{i+1}$ to $\sigma_{i}$ is not simulated yet. See Section 5.2 on definition of the costs for the graph. After failure or reaching the goal of current path, another path is found using $A^{*}$ prune. $A^{*}$ prune will consider the increased cost and already simulated paths, and return next best not-simulated path. Fig $3 d$ illustrates how simulating a new path may result in multiple tree vertices mapping to the same stance graph vertex.

If the low-level controller fails a move, further attempts may benefit from trying the move from slightly different starting poses (remember that many poses and character states may map to the same stance). This is why we check for failures one node ahead towards $\sigma_{i+2}$ on line 11 of Algorithm 1 and re-simulate not only the failed edge, but also the preceding edge when all already existing states have failed in reaching $\sigma_{i+2}$. Fig 4 shows an example of this, where both edges $\sigma_{0} \sigma_{\text {start }}$ and $\sigma_{1} \sigma_{\text {start }}$ have initially failed, and one of them has to be attempted again to reach the goal nodes. Before making a new attempt at $\sigma_{1} \sigma_{\text {start }}$, we also resimulate $\sigma_{0} \sigma_{1}$ to get a new starting state $\mathbf{s}_{2}$ instead of $\mathbf{s}_{1}$.

\subsection{Building the Stance Graph}

We build the stance graph in two steps, and we want to create all possible stance nodes and edges that a set of holds $\mathcal{H}$ can admit, while keeping the graph simple enough to parse. In step 1, we know that the climber is at the free stance $\sigma_{0}$ where its hands and feet are detached from the holds, and we are allowed to go only to $\sigma_{\text {start }}$ which is defined by the user. However, in step 2 , we are in $\sigma_{\text {start }}$ and we are allowed to create any possible stance from $\mathcal{H}$. To do so, given a stance node $\sigma_{n}$ connected to graph, we find a set of candidate neighbors $\sigma_{i}$ around $\sigma_{n}$ such that:

- In step $1, \sigma_{i, j}=\left\{\mathbf{x}_{-1}, \sigma_{\text {start }, j}, \sigma_{n, j}\right\}$ for $j=\{$ lf,rf,lh,rh $\}$.

- In step $2, \sigma_{i, j}=\left\{\mathbf{x}_{-1}, \mathcal{H}_{\sigma}, \sigma_{n, j}\right\}$ for $j=\{$ lf,rf,lh,rh $\}$ where $\mathcal{H}_{\sigma} \subset \mathcal{H}$ and contains all holds satisfying the half-circle distance constraints illustrated in Fig 5.

We recursively add to the stance graph all the candidate neighbors that satisfy the following additional pruning criteria:

(1) If $\sigma_{i}$ is created in step 1 and $\sigma_{n}=\sigma_{0}$ then $\sigma_{i}$ can connect to graph if at least one of the hands is attached to the holds (i.e., the climber must start by first grasping a hand on a start hold).

(2) If $\sigma_{i}$ is created in step 1 and only one hand is connected in $\sigma_{n}$, then $\sigma_{i}$ can connect to the graph when at least one more hand or foot is attached to the holds (i.e, after the first hand, the climber must place the second hand or a foot). 
(3) At most two elements in $\sigma_{n}$ and $\sigma_{i}$ can be different (i.e., move at most two limbs at the same time).

(4) If first or second conditions are not met, then $\sigma_{i}$ can connect to $\sigma_{n}$ if in order to reach $\sigma_{i}$ from $\sigma_{n}$, we always have one of the hands connected and at least two parts of the climber are connected to holds (i.e., we do not consider rare movement that have both hands free or only use a single point of support).

(5) Foot holds cannot be higher than $0.1 \mathrm{~cm}$ above the highest hand hold $\sigma_{i}$. While "heel hooks" and "toe hooks" at close to or equal to hand height are fairly common, it's rare for the feet to be much higher than hands. This heuristic avoids generating unnecessary inverted poses.

(6) If all hands and feet are connected in $\sigma_{i}$, the number of unique holds in $\sigma_{i}$ should be at least three (i.e., only 1 or 2 support points result in unstable balance and is very rare).

(7) Distances of hand to hand, foot to foot and hand to foot in $\sigma_{i}$ should not exceed the maximum distances allowed in climber's body.

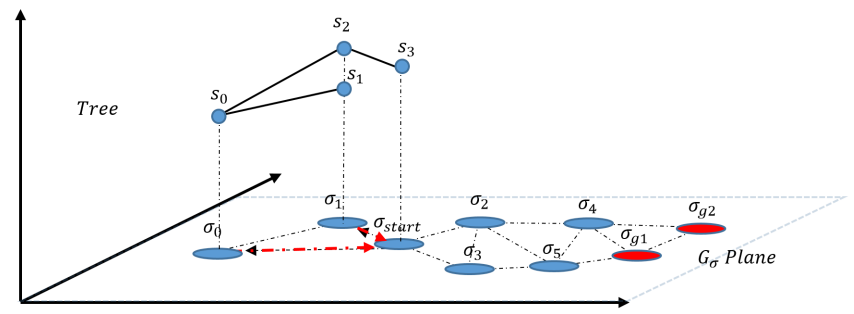

Fig. 4. A rare case where the low-level controller initially fails in simulating both edges $\sigma_{0} \sigma_{\text {start }}$ and $\sigma_{1} \sigma_{\text {start }}$. In this case, edge $\sigma_{0} \sigma_{1}$ gets resimulated, and simulation of $\sigma_{1} \sigma_{\text {start }}$ starts from $\mathbf{s}_{2}$ instead of $\mathbf{s}_{1}$.

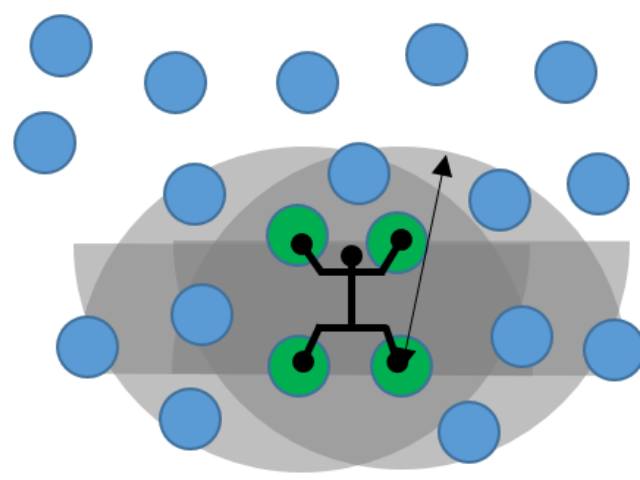

Fig. 5. Determining candidate neighbor stances based on half-circle distance constraints at each hold of the current stance (green color), assuming that at least one hand hold and one foot hold will not change between neighbor stances. The union of the shaded half-circles denotes the holds among which all possible stances can be neighbors of the current stance if they satisfy further pruning criteria. The radius of the half-circles corresponds to the maximum distance between two end-effectors of the climber model.

\section{$5.2 \quad A^{*}$ Prune}

$A^{*}$ prune finds paths as an ordered list of least to highest cost, with the cost of the path computed as the sum of graph edge and node costs. As we do the path finding prior to optimizing the actual movements, we cannot base the edge costs on the torques and forces exerted by the climber. Instead, we formulate the costs as

$$
\operatorname{cost}\left(\sigma_{i}, \sigma_{j}\right)=c_{\text {dyn }}\left(\sigma_{i}, \sigma_{j}\right)+c_{\mathrm{e}}\left(\sigma_{i}, \sigma_{j}\right)+c_{\mathrm{n}}\left(\sigma_{j}\right),
$$

where $c_{\text {dyn }}\left(\sigma_{i}, \sigma_{j}\right)$ denotes a dynamic cost for stance graph edge $\sigma_{i} \sigma_{j}$. The cost is initially set to zero and increased by parameter $c_{\text {fail }}$ when the low-level controller fails to reach $\sigma_{j}$ (line 25 of Algorithm $1)$. As the climber can try a move in both directions, $c_{\text {dyn }}\left(\sigma_{i}, \sigma_{j}\right)$ may differ from $c_{\text {dyn }}\left(\sigma_{j}, \sigma_{i}\right)$.

The $c_{\mathrm{e}}\left(\sigma_{i}, \sigma_{j}\right)$ and $c_{\mathrm{n}}\left(\sigma_{j}\right)$ in Equation 1 are static edge and node costs that represent the difficulty and effort of moving from $\sigma_{i}$ to $\sigma_{j}$. The costs are computed based on various heuristics when the stance graph is built. The following describes the heuristics and related tuning parameters; parameter values used in our simulations are given in Section 6. The heuristics are denoted with $h()$, and tuning parameters are denoted with $c$ for cost values, and $d$ for distances.

The static edge cost $c_{\mathrm{e}}\left(\sigma_{i}, \sigma_{j}\right)$ is composed of three heuristics as

$$
c_{\mathrm{e}}\left(\sigma_{i}, \sigma_{j}\right)=h_{\text {dist }}\left(\sigma_{i}, \sigma_{j}\right)+h_{2 \text { limbs }}\left(\sigma_{i}, \sigma_{j}\right)+h_{\text {unstable }}\left(\sigma_{i}, \sigma_{j}\right) \text {. }
$$

The heuristic $h_{\text {dist }}\left(\sigma_{i}, \sigma_{j}\right)$ makes the planning avoid redundant movement; it equals the sum of distances of holds between the stances. If a limb is free (not attached to a hold) in the source stance, we cannot know the corresponding distance, and instead use a

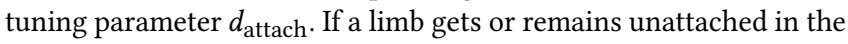
target stance, we use a parameter $d_{\text {free }}$.

The heuristic $h_{2 \operatorname{limbs}}\left(\sigma_{i}, \sigma_{j}\right)$ denotes the difficulty of moving more than one limb at a time. We use a tuning parameter $h_{2 \operatorname{limbs}}\left(\sigma_{i}, \sigma_{j}\right)=$ $c_{2 l i m b s}$ if the climber is moving more than one limb between the stances, and the movement is not considered simple "ladder climbing", which we define as 1) only the opposite hand and leg are moving, 2) moving limbs get attached in the target stance, and 3) the distance between the moving limbs is greater than parameter $d_{\text {too-close }}$ Otherwise, $h_{2 \text { limbs }}\left(\sigma_{i}, \sigma_{j}\right)=0$.

The heuristic $h_{\text {unstable }}\left(\sigma_{i}, \sigma_{j}\right)$ penalizes cases where the number of unique holds connected to hands and feet is less than or equal to two during the move, and the distance between the holds is less

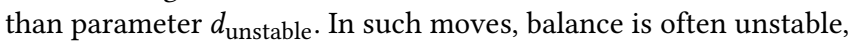
and we use cost parameter $h_{\text {unstable }}\left(\sigma_{i}, \sigma_{j}\right)=c_{\text {unstable }}$. Otherwise, $h_{\text {unstable }}\left(\sigma_{i}, \sigma_{j}\right)=0$.

The static node cost $c_{\mathrm{n}}\left(\sigma_{j}\right)$ of Equation 1 comprises the following heuristics:

$$
c_{\mathrm{n}}\left(\sigma_{j}\right)=h_{\text {free }}\left(\sigma_{j}\right)+h_{\text {match }}\left(\sigma_{j}\right)+h_{\text {crossing }}\left(\sigma_{j}\right)+h_{\text {too-close }}\left(\sigma_{j}\right) .
$$

The heuristic $h_{\text {free }}\left(\sigma_{j}\right)$ is nonzero if the target stance $\sigma_{j}$ has a free hand or foot. A free foot is more preferable, as climbers often use free legs for balancing (e.g., so called "flagging"). We thus use $h_{\text {free }}\left(\sigma_{j}\right)=c_{\text {free-foot }}$ for feet and $h_{\text {free }}\left(\sigma_{j}\right)=c_{\text {free-hand }}$ for hands.

The heuristic $h_{\text {match }}\left(\sigma_{j}\right)=c_{\text {match }}$ if two feet are attached to the same hold, which can be cumbersome in real climbing. Otherwise, $h_{\text {match }}\left(\sigma_{j}\right)=0$. In climbing lingo, "match" means using a single hold for two limbs. 


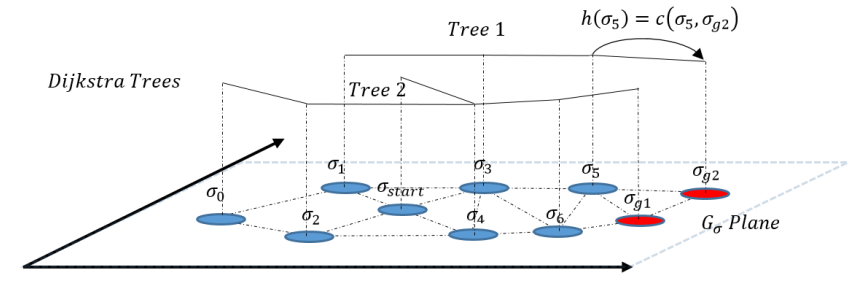

Fig. 6. Shortest path trees created by Dijkstra Algorithm for calculating $h\left(\sigma_{n}\right)$.

The heuristic $h_{\text {crossing }}\left(\sigma_{j}\right)=c_{\text {crossing }}$ if hands or feet are crossed along the $x$ axis, or either of the hands is lower than any of the feet Otherwise, $h_{\text {crossing }}\left(\sigma_{j}\right)=0$.

Heuristic $h_{\text {too-close }}\left(\sigma_{j}\right)=c_{\text {too-close }} \sum_{k} \max \left(0,\left(1-\frac{d_{k}}{d_{\text {too-close }}}\right)\right)$, where the summation is over all hand-foot pairs for the limbs that are attached to holds in stance $\sigma_{j}$, and $d_{k}$ is the distance between the hand and feet hold in pair $k$. In other words, we prefer to keep hands and feet further apart than $d_{\text {too-close }}$ for stable balance.

Running $A^{*}$ prune: As suggested by the original $\mathrm{A}^{*}$ prune paper [Liu and Ramakrishnan 2001], we run Dijkstra's algorithm on the stance graph to compute the sum of the preference heuristics from any graph node to the closest goal stance. Since there are multiple goal nodes, we start Dijkstra backwards starting from all goal nodes on the graph toward the initial $\sigma_{0}$. This produces a number of shortest path trees, as illustrated in Fig 6. Then, as long as there is no failure in following the planned path by the low-level controller, i.e., no increase in edge costs, we can return multiple paths sorted by cost using $A^{*}$ prune without restarting it. When a change in the stance graph happens, we restart $A^{*}$ prune algorithm which means we re-run Dijkstra algorithm to re-calculate the heuristics, and clear the list of all possible paths, but the list of already found paths remains intact.

\subsection{Low-Level Climber Controller}

To grow the tree, i.e., synthesize movements from a starting state to a target stance, we use a sampling-based movement optimization/control approach. The supplemental video shows results generated with both Covariance Matrix Adaptation Evolution Strategy (CMA-ES) [Hansen 2006], a standard technique in the graphics literature, as well as the more recent Control Particle Belief Propagation (C-PBP) method [Hämäläinen et al. 2015]. We use Open Dynamics Engine as our simulator with a time step of $1 / 30$ seconds (30 FPS animation). Both control methods are suitable for model-predictive control using a black-box dynamics simulator. Both methods can be used for offline optimization, although C-PBP was originally demonstrated in receding-horizon online control except for an offline toy example.

In both methods, the optimization process comprises an iteration of 1) sampling control parameters, 2) simulating the corresponding climber state trajectories, 3) evaluating a cost function based on the controls and states, and 4) refining the control sampling distribution based on the costs. As a probabilistic method, C-PBP internally treats the costs as negative logarithms of probability densities which leads to a Gaussian mixture formulation for the sampling distribution. In CMA-ES, the sampling distribution is a single Gaussian.

For each move, we optimize joint motor target velocities over a planning horizon of 1.5 seconds (C-PBP offline) or 0.5-2.0 seconds (CMA-ES). CMA-ES samples two "keyframes", each of which specifies a duration in the range $0.25 \ldots .0 .75$ and a target angular velocity for each joint. The velocities are linearly interpolated (i.e., constant acceleration) to the targets over the durations starting from the angular velocities of the initial simulation state. C-PBP by default samples velocities for each simulation step, but we use the same sampled values for 4 successive simulation steps, i.e., there's a controller step only for every 4 simulation steps. This reduces high-frequency noise while still allowing for enough temporal detail. In all our experiments, we use 64 samples per iteration, except for 256 for the first CMA-ES iteration.

For all methods, we use the following cost function, summed over the simulated movement trajectory:

$$
\begin{aligned}
\operatorname{cost}_{\mathrm{obj}} & =\frac{1}{k_{\sigma}^{2}} \sum_{j} \phi(\mathbf{s}, j)\left\|\mathbf{x}_{j}-\sigma_{i+1, j}\right\|^{2} \\
& +\frac{1}{k_{\text {com }}^{2}}\left\|\widehat{\mathbf{x}}_{\mathrm{com}}-\mathbf{x}_{\mathrm{com}}\right\|^{2} \\
& +\frac{1}{k_{\text {posture }}^{2}} \sum_{i}\left\|\theta_{i}-\widehat{\theta}_{i}\right\|^{2} \\
& +\frac{1}{k_{\text {dir }}^{2}}\left\|\mathbf{d}_{\text {torso }}-\mathbf{d}_{\text {wall }}\right\|^{2} \\
& +\frac{1}{k_{\text {velocity }}^{2}} \sum_{b}\left\|\mathbf{v}_{b}\right\|^{2} \\
& +\frac{1}{k_{\text {force }}^{2}}\left(\sum_{i}\left\|\tau_{i}\right\|^{2}+\sum_{j}\left\|\mathbf{F}_{j}\right\|^{2}\right)
\end{aligned}
$$

where the $k$ :s are tuning parameters corresponding to the following cost components:

(1) Reaching the target stance $\sigma_{i}$ (parameter $k_{\sigma}$ ): This objective helps the low-level controller to get climber's hands and feet from $\mathbf{s}_{\text {init }}$ to desired stance $\sigma_{i+1}$. The summation in this component is over $j=\{\mathrm{lf}, \mathrm{rf}, \mathrm{lh}, \mathrm{rh}\} . \phi(\mathrm{s}, j)$ is 1 for limbs with defined targets, and zero otherwise.

(2) Keeping center of mass close to the wall (parameter $k_{\text {com }}$ ): In general, good climbers tend to maintain postures where they stay close to the wall. $\widehat{\mathbf{x}}_{\text {com }}$ is the closest point of the wall at the same height as $\mathbf{x}_{\text {com }}$, i.e., the cost function component corresponds to squared horizontal distance from the wall.

(3) Keeping a preferred posture (parameter $k_{\text {posture }}$ ): This cost makes the climber prefer postures close to the default posture in Fig 7 (right) with value of $k_{\text {posture }}=k_{\text {posture-climb }}$, except for the times where the character is standing on the ground, where we use the T-pose as the default with the value of $k_{\text {posture }}=k_{\text {posture-free }}$. This helps producing more natural movement and preventing the climber ending up in a state from which it has difficulties to continue. We denote joint angles and their default pose values with $\theta_{i}$ and $\widehat{\theta}_{i}$, respectively. The summation is over all joints. Note that 
when using C-PBP, this cost component is implemented implicitly as a Gaussian component in the sampling proposal, similar to the original paper [Hämäläinen et al. 2015].

(4) Facing towards the wall (parameter $k_{\text {dir }}$ ): This component makes the climber prefer facing the wall, although temporary deviations are allowed. We denote the climber's direction with $\mathbf{d}_{\text {torso }}$ and our desired direction with $\mathbf{d}_{\text {wall }}$.

(5) Prefer calm movements (parameter $k_{\text {velocity }}$ ). This component is not used with CMA-ES, but it helps reducing sampling noise (jittery movement) with C-PBP. We denote each body velocity with $\mathbf{v}_{b}$ where $b$ is the body name. The summation in this component is over all bodies of the humanoid agent.

(6) Minimize applied torques and finger strain $\left(k_{\text {force }}\right.$ param.): This component, i.e., the control cost, is the sum of the squares of applied joint motor torques and the forces exerted by hands on hand holds. We denote the torque around joint angle $i$ by $\tau_{i}$, and force on hand $j$ by $\mathbf{F}_{j}$. Note that although we optimize motor velocities instead of raw torques for better simulation stability, Open Dynamics Engine lets one query the amount of torque the motor needed for reaching the velocity, and the motors also have a maximum possible torque parameter for realism.

Termination Conditions: To simulate forward climber's state from $\mathbf{s}_{i n i t}$ toward $\sigma_{i+1}$ in Algorithm 1, we first detach climber's hands and feet from the holds that differ between the start and end stances, and then run C-PBP or CMA-ES until one of the following termination conditions is satisfied:

- Maximum iterations reached.

- When cost $_{\mathrm{obj}}$ stops reducing.

\section{SIMULATIONS}

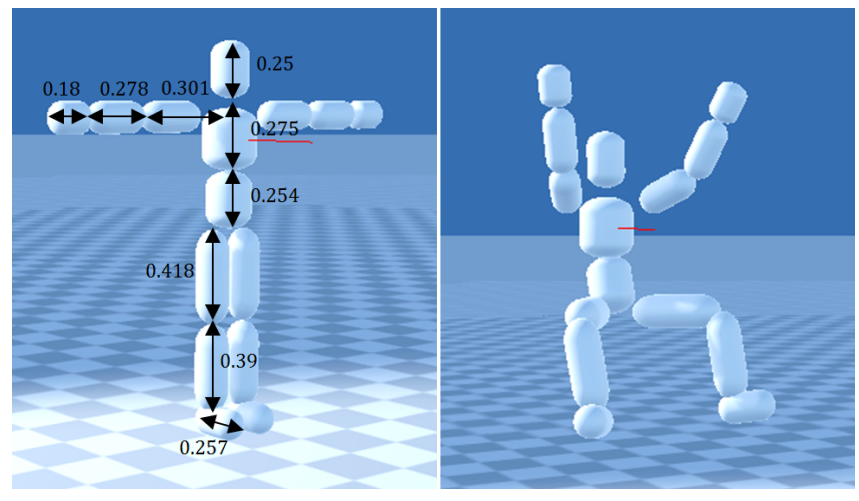

Fig. 7. Left: Climber in T-Pose with measures in meters. Right: Climber's preferred posture.

We have simulated our method on five different scenarios, including three different bouldering routes, a 45 degree tilted wall, and a 10 meter high wall with 48 holds (see supplemental video). Our climber weights $70 \mathrm{~kg}$ and the measures of its body are shown in Fig 7. We let the method find $k=10$ different routes from $\mathbf{s}_{0}$ where the climber is in T-pose on the ground, toward the goal hold $\mathbf{x}_{g}$. When $k$ paths are found, we choose two paths for demonstration on the supplemental video, 1) the one with least amount of limbs moving between holds, and 2) the one with the lowest sum of control costs. On the long wall we only run the method for the first path.

We use the following parameter values: $r_{h}$ and $\theta_{0}$ in Section 4 are set to 0.125 meters and $120^{\circ}$, respectively. $c_{\text {fail }}$ in Algorithm 1 and other edge and node cost parameters of Section 5.2 are shown in Table 1. The low-level movement optimization cost parameters of Section 5.3 are given in Table 2.

Table 1. Parameter values for stance graph edge and node costs

\begin{tabular}{|l|l||l|l|}
\hline Parameter & Value & Parameter & Value \\
\hline \hline$c_{\text {fail }}$ & $10^{5}$ & $c_{\text {crossing }}$ & 100 \\
$c_{\text {free-foot }}$ & 20 & $c_{\text {too-close }}$ & $10^{3}$ \\
$c_{\text {free-hand }}$ & 250 & $c_{\text {2limbs }}$ & 120 \\
$c_{\text {match }}$ & 100 & $c_{\text {unstable }}$ & 500 \\
$d_{\text {too-close }}$ & $0.5 m$ & $d_{\text {attach }}$ & $0.5 m$ \\
$d_{\text {free }}$ & $2 m$ & $d_{\text {unstable }}$ & $0.95 m$ \\
\hline
\end{tabular}

Table 2. Parameter values for low-level controller cost function

\begin{tabular}{|l|l|l|}
\hline Parameter & C-PBP & CMA-ES \\
\hline \hline$k_{\text {dir }}$ & 0.5 & 0.5 \\
\hline$k_{\sigma}$ & $0.01(m)$ & $0.25 * 10^{-2}(m)$ \\
\hline$k_{\text {velocity }}$ & $0.5\left(m / s^{2}\right)$ & $100\left(m / s^{2}\right)$ \\
\hline$k_{\text {posture-free }}$ & $0.087^{\mathrm{rad}}$ & $0.087^{\mathrm{rad}}$ \\
\hline$k_{\text {posture-climb }}$ & $0.785^{\mathrm{rad}}$ & $0.785^{\mathrm{rad}}$ \\
\hline$k_{\text {com }}$ & $20(m)$ & $20(m)$ \\
\hline$k_{\text {force }}$ & $250(N)$ & $250(N)$ \\
\hline
\end{tabular}

\subsection{Evaluation}

As illustrated by Fig 1 and the supplemental video, our system is able to find plausible solutions to all the simulated problems. On the bouldering routes, finding the first path takes around one minute of CPU time on Intel(R) Xeon(R) CPU E3-1231 v3 @ 3.40 GHz. The sampling-based movement optimization may sometimes fail, but the method is then able to find alternative paths. The cost of building the stance graph is low compared to the planning; graph building only takes a few seconds on the bouldering routes. The high wall naturally takes longer to process, but our system was nevertheless able to find a path in 13 and 3.2 minutes CPU time with CMA-ES and C-PBP as the low-level controller, respectively. Building the stance graph for the high wall takes around one minute.

CMA-ES and C-PBP have both their advantages and disadvantages. When we have shown the results to experienced climbers, they comment that CMA-ES produces more determined and skilled movement, while C-PBP looks more like a hobbyist who goes to the bouldering gym once a week. We agree, and attribute this difference 
mostly to the number of controlled degrees of freedom. CMA-ES only optimizes a piecewise linear velocity curve with two control points, which completely removes the movement noise generated by C-PBP. On the other hand, some less advanced climbers have commented that C-PBP results are more natural, as human movement usually does have imperfections. C-PBP noise can also be reduced with increased sampling budget. When we tried to achieve more natural movement by increasing the number of CMA-ES control points (thus increasing the number of optimized parameters), we could not get the optimization to converge anymore. C-PBP due to its dynamic programming nature is at least in theory less prone to the curse of dimensionality with regards to the number of planning steps.

Regarding computing cost, CMA-ES needs more iterations with similar sampling budget due to oscillation around the final cost minimum found. This is illustrated in the supplemental video; while optimizing each move, we visualize both the starting pose and the end pose of the best sample of the current optimization iteration. Due to the slow convergence, CMA-ES typically needs about twice the CPU time to find a path. On the other hand, CMA-ES with our settings is more reliable in terms of avoiding greedy behaviors leading to failure, illustrated in Fig 8. We ran 20 simulations on following the first route planned by $\mathrm{A}^{*}$ prune in the bouldering route of Fig 8 for both optimization methods with 4 failure cases for CMA-ES and 11 failure cases for C-PBP (offline). The greediness of C-PBP can be adjusted with the resampling threshold parameter, but it appears difficult to tune. We have run all our tests with the default 0.5 ; convergence degraded rapidly when we tried adjusting the parameter.

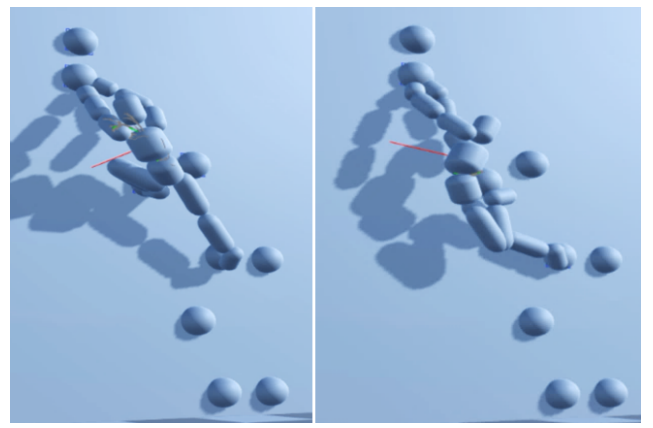

Fig. 8. Low-level controller success (left) and failure (right) cases. In the failure cases, the left foot reaches for a hold too greedily, trying to go behind the climber's back. We observed such failures more often with C-PBP than CMA-ES.

The maximum torque allowed for the joint motors can be easily adjusted to simulate stronger or weaker climbers. Weakening the agent increases the average time needed to find a route, as many of the tried paths fail; qualitatively, the successful climbs exhibit more calm and conservative movements. We demonstrate a comparison between weak and strong climber's movements on the supplemental video at 1:35.

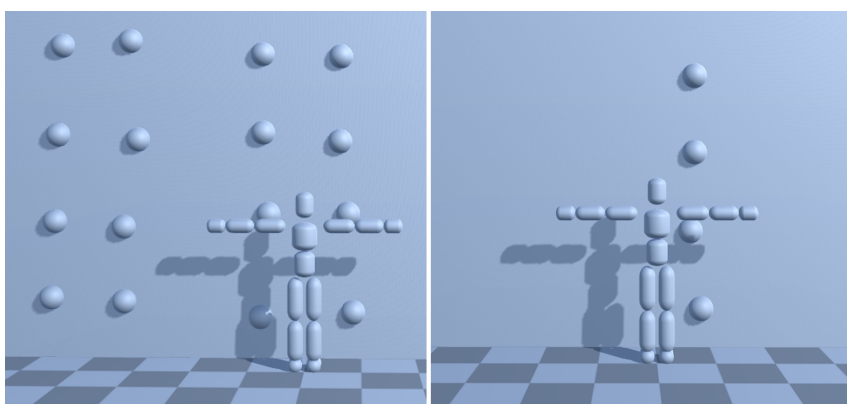

Fig. 9. Two examples of scalability test cases using grids of holds with some random deviation added to the hold positions.

\subsection{Scalability}

In order to evaluate the scalability of our method, we created different sizes of hold grids, as illustrated in Fig 9. The start and goal holds were placed in opposite corners, requiring the character to traverse the wall both horizontally and vertically. The holds were also randomly displaced from the grid to make the test more realistic. We tested configurations with $1 . . .4$ columns and $2 \ldots 8$ rows of holds (climbing just one row does not make sense).

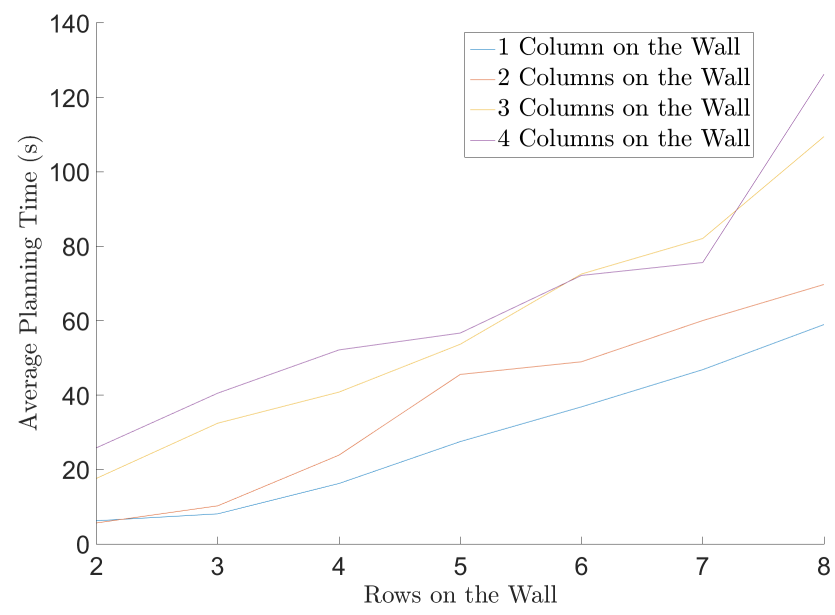

Fig. 10. Average time needed to plan and simulate 10 paths as a function of climbing wall size, using the hold grids of Fig 9.

Fig 10 shows the CPU time of planning and simulating 10 paths with different wall sizes, averaged over 6 simulations, each with different random hold displacements. Time was measured on an Intel(R) Xeon(R) CPU E3-1231 v3 @ 3.40 GHz. Fig 11 shows the corresponding stance graph sizes. The data indicates that with such hold grids, both graph size and the planning and simulation time grow roughly linearly as a function of wall height. The linear slope of graph size is steeper with more hold columns. 


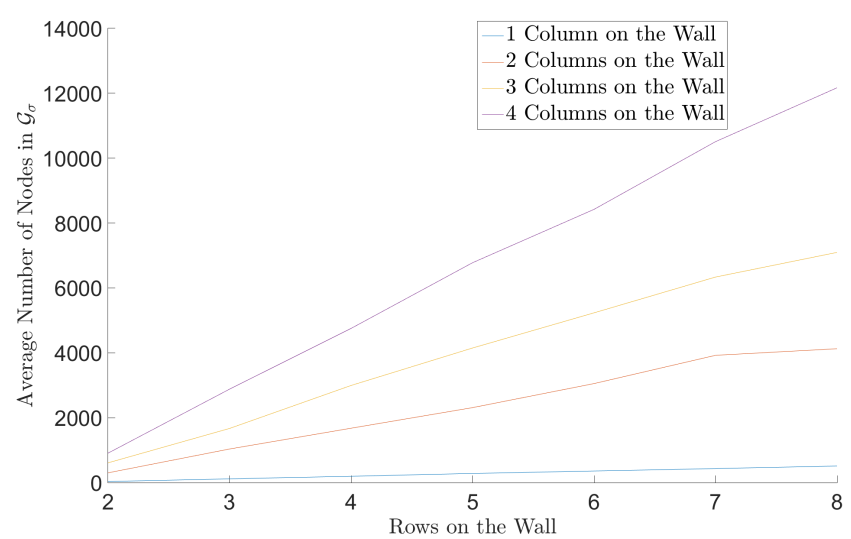

Fig. 11. Average number of nodes in the stance graph as a function of climbing wall size, using the hold grids of Fig 9.

\section{APPLICATIONS}

We consider sports coaching and computer-aided climbing route design/verification (in both virtual and real worlds) as possible applications for our system. Climbing is a rapidly growing sport that has recently been approved for the 2020 Olympics. Indoor climbing environments are also highly suitable for technological augmentation, e.g. [Aladdin and Kry 2012; Kajastila et al. 2016]; we are presently preparing a user study that measures the effectiveness of our system in helping novice climbers in Augmented Reality climbing. We also believe our approach can be generalized to climbing more freeform shapes without holds, provided that key features of the environment such as graspable edges are discretized as sets of 'virtual holds' when building the stance graph.

\section{LIMITATIONS AND FUTURE WORK}

Although this paper does advance the state of the art in climbing movement planning and synthesis, we must acknowledge some limitations that we intend to address in future work. First, our heuristics for both graph connectivity (Section 5.1) and edge costs (Section 5.2) could probably be simplified. On the other hand, there's only so much one can do with hand-tuned heuristics; having shown that the stance graph approach is feasible, we intend to learn the connectivity and costs from data in the future. Our present system can act as a powerful exploration and data generation tool, training a machine learning predictor of stance-to-stance climbing move difficulty, required torques, and flexibility. Such a predictor could then be used both in the graph building and path finding, increasing the probability of finding and simulating relevant and interesting paths. In a similar manner, we should be able to train faster low-level controllers based on the outputs of CMA-ES or C-PBP, or at least learn good initial guesses for transitioning from a given state and stance to a target stance.

A second limitation of our system is the simulation of the holds as ball-and-socket joints. This amounts to having all the simulated holds as what climbers call "jugs", i.e., large holds with a cavity to place one's fingers or toes in. In reality, a hold's shape determines how much one can pull the hold in all directions without slipping, and learning to predict this and plan climbing strategy accordingly is a big part of the sport. In the future, we intend to simulate this by defining a directionally varying maximum forces that the hold joints tolerate.

A further limitation is that our low-level controller only considers one stance graph edge at a time. This means that even if our character can transition between stances, the resulting pose and movement state may not be optimal for continuing towards the next stance of the path. We in effect assume that if a different intermediate pose is required, the low-level controller can generate it as part of the next synthesized movement trajectory. Although our current handling of low-level controller failures and multiple poses per stance (Figs 3 and 4) appears sufficient in our test cases, we will attempt to optimize movements more than one edge at a time in future work.

\section{CONCLUSION}

We have proposed and demonstrated a novel approach to planning and synthesizing physically-based humanoid wall climbing movements. We combine a high-level graph-based path planner with a low-level controller/optimizer to synthesize plausible climbing motions. Our high-level path planner utilizes the concept of stances or assignments of holds for the climber's hands and feet, and builds a graph in the low-dimensional stance space. Paths are then found and simulated using the low-level controller, eventually growing a tree in the climber's high-dimensional state space, which gives multiple plausible solutions for navigating from a start stance to a goal stance with physically based simulated movements. As a climbing-specific adaptation of this general approach, we have formulated heuristics for both restricting the graph's connectivity, and guiding the pathfinding. We don't claim that the heuristics are optimal; what is noteworthy is that they allowed us to demonstrate the feasibility of the graph search approach. In future work we intend to learn predictors of graph connectivity and edge costs from data generated by our system, running it on a large enough training set of randomly generated routes.

We have demonstrated results using two different methods, CPBP and CMA-ES, for the low-level movement synthesis. Both methods were able to generate plausible movements, although performance and movement quality varies. A natural extension that we currently pursue is using the present optimization for training faster and better controllers.

\section{ACKNOWLEDGEMENTS}

We thank our reviewers for their highly valuable feedback. This work has been supported by Academy of Finland and the Finnish Funding Agency for Innovation.

\section{REFERENCES}

Rami Aladdin and Paul Kry. 2012. Static Pose Reconstruction with an Instrumented Bouldering Wall. In Proceedings of the 18th ACM Symposium on Virtual Reality Software and Technology (VRST '12). ACM, New York, NY, USA, 177-184. DOI: https://doi.org/10.1145/2407336.2407369

Timothy Bretl. 2006. Motion Planning of Multi-Limbed Robots Subject to Equilibrium Constraints: The Free-Climbing Robot Problem. The International fournal of Robotics Research 25, 4 (2006), 317-342 
Oliver Brock and Oussama Khatib. 2002. Elastic Strips: A Framework for Motion Generation in Human Environments. The International fournal of Robotics Research 21, 12 (2002), 1031-1052.

Myung Geol Choi, Manmyung Kim, Kyung Lyul Hyun, and Jehee Lee. 2011. Deformable Motion: Squeezing into Cluttered Environments. In Computer Graphics Forum, Vol. 30. Wiley Online Library, 445-453.

Min Gyu Choi, Jehee Lee, and Sung Yong Shin. 2003. Planning Biped Locomotion Using Motion Capture Data and Probabilistic Roadmaps. ACM Transactions on Graphics (TOG) 22, 2 (2003), 182-203.

Nguyen Anh Dung and Akira Shimada. 2014. A Path-Planning Algorithm for Humanoid Climbing Robot Using Kinect Sensor. In SICE Annual Conference (SICE) in Sapporo. IEEE, 1549-1554. DOI : https://doi.org/10.1109/SICE.2014.6935284

David Eppstein. 1998. Finding the k Shortest Paths. SIAM Journal on Computing 28, 2 (1998), 652-673.

David Eppstein and others. 2016. k-Best Enumeration. Springer. (2016). DOI : https //doi.org/10.1007/978-3-642-27848-8_733-1

Ian Garcia and Jonathan P How. 2005. Improving the Efficiency of Rapidly-Exploring Random Trees Using a Potential Function Planner. In Proceedings of the 44th IEEE Conference on Decision and Control. IEEE, 7965-7970.

Jürgen Guldner, Vadim I Utkin, and Hideki Hashimoto. 1997. Robot Obstacle Avoidance in n-Dimensional Space Using Planar Harmonic Artificial Potential Fields. Fournal of Dynamic Systems, Measurement, and Control 119, 2 (1997), 160-166.

Perttu Hämäläinen, Joose Rajamäki, and C Karen Liu. 2015. Online Control of Simulated Humanoids Using Particle Belief Propagation. ACM Transactions on Graphics (TOG) 34,4 (2015), 81.

Nikolaus Hansen. 2006. The CMA Evolution Strategy: A Comparing Review. In Towards a New Evolutionary Computation. Springer, 75-102.

Michael Hoy, Alexey S Matveev, and Andrey V Savkin. 2015. Algorithms for CollisionFree Navigation of Mobile Robots in Complex Cluttered Environments: A Survey. Robotica 33, 03 (2015), 463-497. DOI : https://doi.org/10.1017/S0263574714000289

Sumit Jain, Yuting Ye, and C Karen Liu. 2009. Optimization-Based Interactive Motion Synthesis. ACM Transactions on Graphics (TOG) 28, 1 (2009), 10.

Raine Kajastila, Leo Holsti, and Perttu Hämäläinen. 2016. The Augmented Climbing Wall: High-Exertion Proximity Interaction on a Wall-Sized Interactive Surface. In Proceedings of the $2016 \mathrm{CHI}$ Conference on Human Factors in Computing Systems (CHI '16). ACM, New York, NY, USA, 758-769. DOI : https://doi.org/10.1145/2858036. 2858450

Lydia E Kavraki, Petr Svestka, J-C Latombe, and Mark H Overmars. 1996. Probabilistic Roadmaps for Path Planning in High-Dimensional Configuration Spaces. IEEE transactions on Robotics and Automation 12, 4 (1996), 566-580.

James J Kuffner and Steven M LaValle. 2000. RRT-Connect: An Efficient Approach to Single-Query Path Planning. In Robotics and Automation, 2000. Proceedings. ICRA'00. IEEE International Conference on, Vol. 2. IEEE, 995-1001.

Steven M. Lavalle. 1998. Rapidly-Exploring Random Trees: A New Tool for Path Planning. Technical Report. TR 98-11, Computer Science Dept., Iowa State Univ. http:// citeseerx.ist.psu.edu/viewdoc/summary?doi=10.1.1.35.1853

Steven M LaValle and James J Kuffner. 2001. Randomized Kinodynamic Planning. The International fournal of Robotics Research 20, 5 (2001), 378-400.

Benoît Libeau, Alain Micaelli, and Olivier Sigaud. 2009. Transfer of Knowledge for a Climbing Virtual Human: A Reinforcement Learning Approach. In Robotics and Automation, 2009. ICRA'09. IEEE International Conference on. IEEE, 2119-2124.

Maxim Likhachev, David I Ferguson, Geoffrey J Gordon, Anthony Stentz, and Sebastian Thrun. 2005. Anytime Dynamic A*: An Anytime, Replanning Algorithm. In ICAPS. $262-271$.

Gang Liu and KG Ramakrishnan. 2001. A* Prune: An Algorithm for Finding K Shortest Paths Subject to Multiple Constraints. In INFOCOM 2001. Twentieth Annual foint Conference of the IEEE Computer and Communications Societies. Proceedings. IEEE, Vol. 2. IEEE, 743-749.

Igor Mordatch, Emanuel Todorov, and Zoran Popović. 2012. Discovery of Complex Behaviors through Contact-Invariant Optimization. ACM Transactions on Graphics (TOG) 31, 4 (2012), 43. DOI : https://doi.org/10.1145/2185520.2185539

Giacomo Nannicini and Leo Liberti. 2008. Shortest Paths on Dynamic Graphs. International Transactions in Operational Research 15, 5 (2008), 551-563.

Tim Olsen, Sheldon Andrews, and Paul Kry. 2014. Computational Climbing for PhysicsBased Characters. Technical Report. The ACM SIGGRAPH / Eurographics Symposium on Computer Animation (SCA posters) . http://cs.mcgill.ca/ sandre17/ climbing/

Mohammad Naim Rastgoo, Bahareh Nakisa, Mohammad Faidzul Nasrudin, Ahmad Nazri, and Mohd Zakree. 2014. A Critical Evaluation of Literature on Robot Path Planning in Dynamic Environment. fournal Of Theoretical And Applied Information Technology 10th 70, 1 (December 2014), 175-185.

Liam Roditty and Uri Zwick. 2004. On Dynamic Shortest Paths Problems. In European Symposium on Algorithms. Springer, 580-591.

F. Sibella, I. Frosio, F. Schena, and N.A. Borghese. 2007. 3D Analysis of the Body Center of Mass in Rock Climbing. Human Movement Science 26, 6 (2007), 841 - 852. DOI : https://doi.org/10.1016/j.humov.2007.05.008
Herbert G Tanner and Amit Kumar. 2005. Towards Decentralization of Multi-Robot Navigation Functions. In Proceedings of the 2005 IEEE International Conference on Robotics and Automation. IEEE, 4132-4137.

Yuval Tassa, Nicolas Mansard, and Emo Todorov. 2014. Control-Limited Differential Dynamic Programming. In Proc. ICRA 2014. IEEE, 1168-1175. DOI : https://doi.org/ 10.1109/ICRA.2014.6907001

Steve Tonneau, Julien Pettré, and Franck Multon. 2014. Task Efficient Contact Configurations for Arbitrary Virtual Creatures. In Proceedings of Graphics Interface 2014 (GI '14). Canadian Information Processing Society, Toronto, Ont., Canada, Canada, 9-16. http://dl.acm.org/citation.cfm?id=2619648.2619652

Dustin J Webb and Jur van den Berg. 2013. Kinodynamic RRT*: Asymptotically Optimal Motion Planning for Robots with Linear Dynamics. In Robotics and Automation (ICRA), 2013 IEEE International Conference on. IEEE, 5054-5061. DOI : https://doi.org/ 10.1109/ICRA.2013.6631299

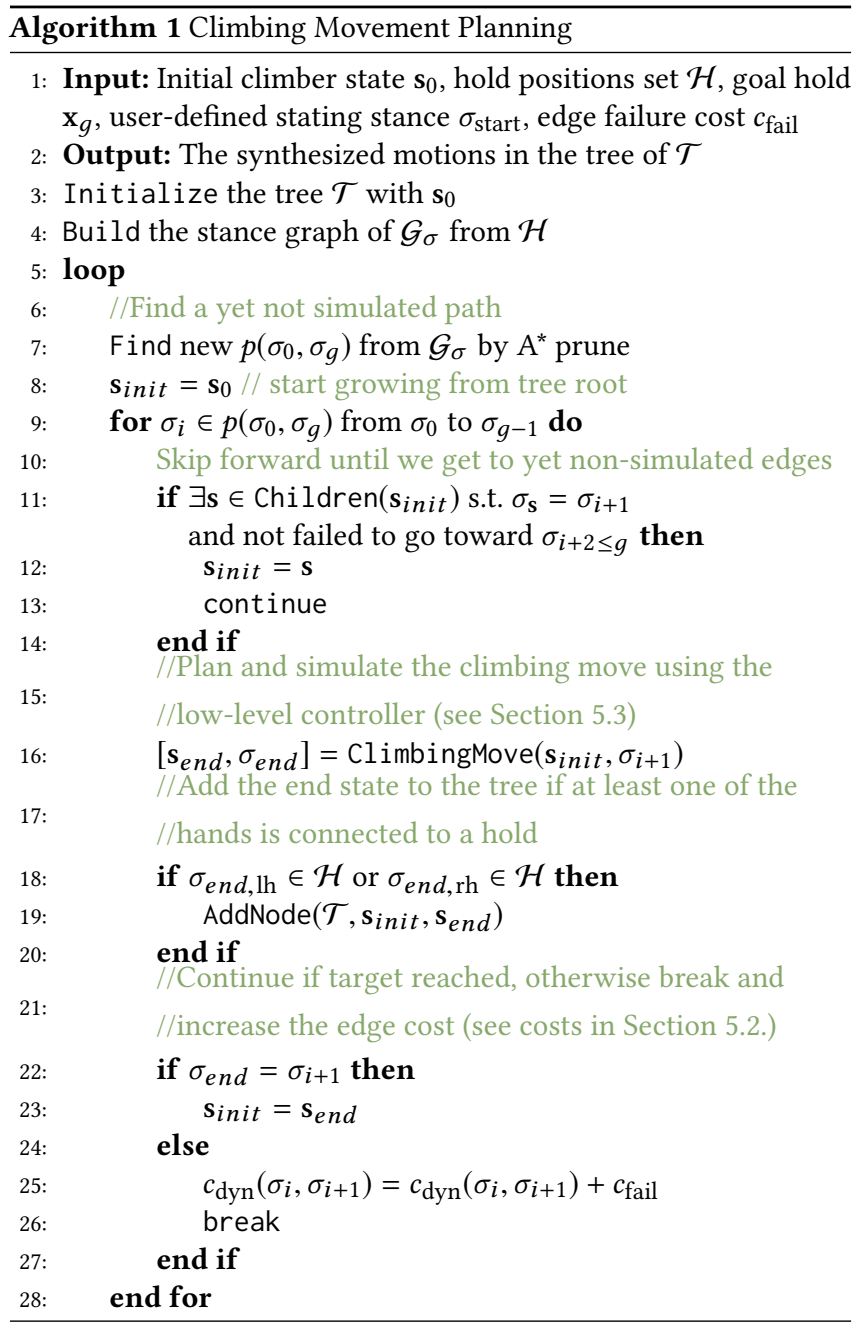

